\title{
Interventional Radiology: Interventions and Techniques in Trauma
}

\author{
Andres Garza-Berlanga ${ }^{1}$. Jorge Lopera ${ }^{1}$
}

Published online: 3 August 2016

(C) Springer International Publishing AG 2016

\begin{abstract}
Purpose of Review The purpose of the review was to describe how interventional radiology procedure contribute in the management of the trauma patient, distinguish the situations where evidence has demonstrated improved outcomes with its use, acknowledge the limitations and controversies of the techniques and their place on management algorithms, and mention some particular situations where, despite lack of evidence, the procedures are commonly employed.

Recent Findings CT seems to be a better indicator of significant vascular injury with the associated high risks when compared to a discordant negative conventional angiogram. Empiric embolization of the injured segments might improve outcomes in these settings. Finding a subcapsular splenic hematoma in $\mathrm{CT}$ is an independent risk factor associated with high rates of NOM failure. Prophylactic interventions are recommended, even in low grade splenic injuries, when a subcapsular splenic hematoma is present. In liver trauma, the injured liver is more susceptible to ischemic injury from arterial embolization with subsequent infarct, biloma, and abscess formation. When needed, angio-embolization should be performed as selective as possible. Subsequent surveillance for ischemic liver injury complications should be instated and, if required, timely therapeutic interventions considered.
\end{abstract}

This article is part of the Topical Collection on Minimally Invasive Surgical Techniques for Trauma

Andres Garza-Berlanga

GarzaBerlang@uthscsa.edu

1 Vascular and Interventional Radiology, Radiology Department, University of Texas Health Science Center San Antonio,

San Antonio, TX, USA
Summary The initial CT scan findings of "contrast blush" and high-grade solid organ injury are some of the best early predictors for failure of the non-operative management (NOM) in the trauma patient. Endovascular interventions improve the outcomes of NOM when clinical or imaging findings indicate a high risk for continued or delayed hemorrhage. Angioembolization improves the outcomes of unstable hemorrhagic pelvic fractures and is useful as complement of damage control surgeries or when the surgical interventions fail to control vascular injuries.

Keywords Interventional radiology $\cdot$ Trauma $\cdot$ Non-operative management $\cdot \mathrm{NOM} \cdot$ Vascular trauma

\section{Introduction}

The severity of vascular trauma range from partial disruptions like intimal tears and dissection flaps to lacerations and complete transections. Vascular injury causes major morbidity and mortality in trauma. Catastrophes can come in the form of hemorrhage or infarction of the supplied tissues.

The availability, velocity and resolution of multi-detector CT scans have improved the diagnostic efficacy in trauma. Whole body scans can be performed within minutes. Sites of injury and hemorrhage can be identified faster and with great accuracy. As a result, trauma centers have changed their evaluation and management protocols. The diagnostic tests like FAST ultrasound, diagnostic paracentesis, and conventional diagnostic angiography have been replaced by CT scans for the most part. If a patient responds favorably to the initial resuscitation efforts, an exploratory laparotomy can be postponed to allow a CT scan evaluation. The ability of fast detection, accurate localization, and good resolution to grade injury has allowed timely use of less invasive, better targeted, 
or more effective interventions, many times waiving the exploratory laparotomy.

CT imaging boosts the success of endovascular therapies by guiding injury localization. By angiography, the vascular injury detection threshold improves tremendously the closer the catheter is placed to the affected vascular segment, for the so called "selective" angiogram. Experimental models estimate that extravasation rates of over $1 \mathrm{~cm}^{3} / \mathrm{min}$ are required to detect hemorrhage by non-selective angiography, when the catheter is in a location more than $10 \mathrm{~cm}$ from the injured segment. On the other hand, in "super-selective" angiography, where the angiogram contrast injection is performed within 1 or $2 \mathrm{~cm}$ of the injury site, the threshold rate to detect extravasation is of less than $0.1 \mathrm{~cm}^{3} / \min [1 \bullet]$.

Endovascular interventions have gained wider acceptance in trauma, sometimes due to its effectives, like in pelvic or retroperitoneal injuries, where surgical localization and control can be difficult. Sometimes, the endovascular approach offers a less invasive option, while maintaining organ function, like in limited spleen or renal injuries. Other times, it is a way to prophylactically treat patients with findings associated the risk of delayed adverse events, such as rupture and hemorrhage from pseudoaneurysms or arteriovenous fistulas.

\section{Splenic Injury}

Splenectomy was the most common treatment for splenic injuries; however, over the last three decades, this practice has changed significantly. The alternative to splenectomy, socalled non-operative management (NOM), consists of medical management, observation and, if required, selective angiography and endovascular treatments. NOM proved to be safe and less morbid than splenectomy in adequately selected patients. It has evolved as the preferred modality for the patients that can endure it. However, failure of NOM with a delayed surgical intervention is related to increased morbi-mortality [2•].

The reported overall failure rates for NOM in splenic injury is in the order of $10 \%\left[3^{\circ}\right.$ ], but varied from 35 to $5 \%$ throughout the evolution of the modality $[3 \cdot, 4]$. NOM failure usually results from continued or delayed hemorrhage at the injury site or from unrecognized concomitant injuries that require surgical intervention such as hollow viscus injury.

The role of endovascular intervention has been to reduce the risk of NOM failure from continued or delayed hemorrhage. Although no consistent evidence-based selection criteria exist to direct angio-embolization, findings of highgrade organ injury, large hemoperitoneum or vascular injury have typically been cited $[5 \bullet, 6 \bullet, 7,8]$. Since the mid 1990s, the presence of "contrast blush" on CT was noted to be a strong predictor of NOM failure [9]. Contrast blush on imaging alludes to the presence of a localized high-density region within a hematoma or injured tissue. It highly correlates with the existence of active extravasation or pseudoaneurysm on angiogram. In blunt spleen trauma, contrast blush is associated with 20 times greater likelihood of NOM failure if managed with observation alone. The "Eastern Association for the Surgery of Trauma practice management guidelines" report a level II recommendation to use angiogram and embolization if NOM is attempted in the presence of contrast blush [10]. Recent cohort reviews support significant benefits of angiography and embolization in the presence of "contrast blush," reducing the NOM failure rates from over $70 \%$ to less than $10 \%$ [8]. More recently, subcapsular splenic hematoma has also been associated with high risk of NOM failure [3•]. Additionally, patients with coagulopathy are frequently managed with a low threshold for undergoing angiogram evaluation. Patients with delayed discrete findings of continuing hemorrhage, such as progressive abdominal pain, decreasing hematocrit, hemodynamic deterioration or persistent hypoperfusion parameters such as acidosis or anuria, are frequently evaluated for angio-embolizaton if immediate surgical intervention is not warranted.

In regards to the radiologic grade of injury, the risk of NOM failure rises in relation to the grade of spleen injury. Reported rates of NOM failure for grade III splenic injuries are around $20 \%, 33 \%$ for grade IV, and $75 \%$ for grade V. Recent cohort studies report a reduced failure trend to as low $5 \%$ when angiogram and embolization are performed routinely for high-grade spleen injuries (III-V). Specifically, the failure risk is reduced to $8 \%$ for grade III injuries, and $20 \%$ for grade IV injuries. There is a lack of information regarding the benefits of embolization in grade $\mathrm{V}$ injuries. The numbers of grade $\mathrm{V}$ injuries managed with NOM in most trauma registries are scant since most require surgical management. Controversy remains, however, on whether NOM should be attempted with grade $\mathrm{V}$ injuries given the high failure rates and the lack of evidence of benefit from embolization [8]. Having said this, most authors recommend routine angioembolization when NOM is intended for high-grade splenic injuries (III-V) $[3 \cdot, 5 \cdot, 8]$.

Regarding subcapsular hematomas, they have recently been identified as an independent risk factor for NOM failure. Subscapular hematomas have NOM failure rates approaching $30 \%$, even in patients with low-grade splenic injuries (I to III). In patients with high-grade (IV) injuries, despite angiogram and embolization in the management, subcapsular hematomas have been associated with NOM failure rates of $80 \%$. Because of this, authors consider that splenectomy might be the best management in cases of grade IV-V injuries with subcapsular hematomas [3•].

Debate remains about the best technique for embolotherapy. Most interventionalists seem to favor subselective embolization when possible [11]. Others argue benefit from combining the subselective distal embolization with a proximal splenic artery occlusion [12•]. On the other hand, some believe that a proximal splenic artery occlusion by 
itself is better, arguing equal efficacy with lower risks of complications $[13,14]$.

Another dilemma is how to proceed when the angiogram fails to localize the injury noted on CT. Evidence indicate that these "angiogram-CT discrepancies" most likely represent false negative angiograms with more than double the risk of NOM failure if left untreated. For these cases, there is a suggestion of benefit with empiric embolization of the injured segments of spleen using the CT images and other secondary discrete angiographic findings like parenchymal enhancement defects that correlate with the laceration site and discrete vessel irregularities to guide vessel selection for treatment [6•].

The risk of complications after angiography and embolization as adjuvant to NOM in the spleen are minimal. Although areas of splenic infarcts are observed on subsequent imaging studies in about $20 \%$ of embolizations, these are usually clinically silent, without apparent immune dysfunction [5•, 15]. Infections and abscesses are occasionally seen [16].

\section{Hepatic Injury}

Liver injury in blunt and penetrating abdominal trauma is relatively common with an incidence of about $25 \%$. Mortality correlates with the grade of liver injury with rates ranging between 5 and $50 \%[2 \bullet, 17]$. NOM has become the preferred option when the situation allows. In contrast to the kidney or the spleen, NOM has good outcomes even with grade V injuries. Surgical interventions are reserved for hemodynamically unstable patients, and patients with peritonitis or concomitant injuries that demand laparotomy. NOM is intended in around $65 \%$ of the blunt liver injury cases with success rates of about $90 \%[2 \bullet, 17]$. For penetrating trauma, NOM is intended in about $30 \%$ of cases with success rates of around $80 \%[18,19]$. Endovascular interventions are indicated to aid NOM when clinical or imaging findings suggest persistent liver hemorrhage [7]. Even for surgical cases, about $30 \%$ will eventually require endovascular interventions to aid with liver vascular injury control [2•, 17, 20•].

The liver has sufficient arterial collateral flow to compromise the efficacy of embolotherapy if both distal and proximal vascular control to the site of injury cannot be achieved [21]. The frequent contracted and spastic vascular conditions in trauma can make distal cannulation challenging. Gelfoam slurry and liquid embolics (glue) are frequently employed from a proximal catheter position in order to obtain the distal control. Proximal control might be secured with selective, more permanent coil embolizations, particularly if concerned with early vessel recanalization and re-bleeds when larger branches are involved.

As with the spleen, CT blush in the liver seems to be a more reliable indicator of the existence of a significant vascular injury than the angiogram (CT-angiogram discrepancy). Failure of the angiogram to identify the liver vascular injury results in doubled risk for NOM failure if left untreated. It has been suggested that empirical selective embolization using the CT images to guide segment selection might be of benefit [6•]. This being said, liver embolotherapy should be performed as selective as possible. In normal conditions, the liver dual blood supply by the portal vein and the hepatic artery makes the organ somewhat resistant to ischemic injury by single arterial occlusion. However, in trauma, there seems to be higher rates of ischemic injury and liver infarcts after angioembolization, particularly in high-grade injuries, presumably as a result of associated compromised porto-venous flow [20•, 22•]. Disproportional elevation of liver transaminases immediately after angio-embolization (100-fold increase) indicates severe ischemic liver injury. Liver necrosis might eventually require further interventions as bilomas and de-vascularized tissues are prone to infection $[2 \bullet, 22 \bullet, 23]$.

\section{Renal Injury}

In abdominal trauma, injury to the kidney or its vasculature presents with an incidence of about 5-7 \%. Most cases are low-grade injuries that can be treated with NOM. Endovascular interventions are indicated to aid NOM in patients with clinical or imaging findings concerning for possible active or intermittent hemorrhage, with as much nephron sparing as possible [24].

Renal artery branching is usually end-organ without significant arterial collateral complementation. Occlusion of a segment results in a wedge infarct of the vascular territory supplied by the branch. Embolotherapy as close as possible to the site of injury results in good vascular control in over $90 \%$ of cases. The angio-embolization technique requires the skills to adequately identify the injured branches, cannulate "superselectively" and occlude the vessels without compromising unnecessary territories. Angio-embolization has the capacity to preserve as much glomeruli as possible from the conserved renal vascular territories [25].

Patients with shattered kidney or pedicle injury (grade V injuries) remain surgical for the most part. Except for a few case reports including successful transection recanalization of the main renal artery limited to $1-2 \mathrm{~h}$ post trauma, most patients with grade $\mathrm{V}$ injuries will have bad outcomes with NOM [24, 25].

\section{Great Vessel Injury}

Injuries of the thoracic aorta remain highly lethal. Victims rarely make it long enough to allow therapeutic interventions attempts.

The thoraco-abdominal contrast-enhanced CT has replaced conventional angiogram as the standard diagnostic test. Conventional angiography is usually reserved for thoracic endovascular aortic repair (TEVAR). 
TEVAR has gained acceptance due to its high technical success rates and relative low morbi-mortality when compared to surgery $[26,27]$. The TEVAR-reported rates of paraplegia are as low as $0-4 \%$ [28]. The great limitation of TEVAR is the limited short-notice shelf availability of proper stent-graft sizes and the necessary ancillary components, particularly in cases of young trauma patients, as these stentgrafts are usually supplied for the treatment of aneurysm or dissection diseases of older patients with larger vessels. In general, endo-graft sizing requires exactitude to avoid oversize graft pleating that can result in collapse, or graft undersize that results in migration or persistent leaks.

Stent-graft landing also requires precision to obtain proper seal and stability to exclude the injured segment of the vessel while avoiding unnecessary over coverage of vital aortic branches. When possible, the left subclavian artery origin should be spared of TEVAR occlusion; however, it has been covered successfully without neurologic compromise when the right vertebral artery is patent $[27,29]$. Otherwise, a second arterial access and wire through the left upper extremity and across the left subclavian artery origin can be used when there is little room for error in graft deployment. This allows accurate localization and possible recanalization maneuvers if the branch of origin was to be unintentionally covered.

For the abdominal aorta and its main visceral branches, penetrating trauma is the most common cause of injury. Management is predominantly surgical, although endovascular interventions can be used in selected cases.

\section{Musculo-skeletal Vessel Injury}

The overall mortality of patients with pelvic fractures is around $15 \%$ but becomes as high as $50 \%$ when they are associated with hemodynamic instability $[30 \bullet, 31]$. Hemorrhage can be from arterial, venous, or osseous origins. Bleeding from a venous source is very common; however, unstable and lethal cases are usually from arterial injury $[32 \cdot, 33 \bullet, 34]$. A broken pelvic osseous ring allows expansion of tissues to blood volume deposition and impairs hemostasis by ineffective compartmental tamponade and clotting factor consumption [35]. Hemorrhage should be addressed without delays along with the resuscitating efforts to avoid getting into the so-called lethal triad of coagulopathy, hypothermia, and acidosis.

Identifying the bleeding source is key. Conventional angiography seems to be more effective than surgical exploration, as hemorrhage frequently derives from multiple arterial branches $[32 \cdot 36]$. Overall, endovascular intervention can effectively control hemorrhagic pelvic fractures in about $90 \%$ of the cases [30॰].

Whenever possible, subselective embolization of the affected vessels should be intended. Awareness of the vascular anatomy and its variations is imperative. The branches of the internal iliac artery are the most commonly affected vessels, particularly the superior gluteal, internal pudendal, and obturator arteries. However, the pelvis counts with a very rich arterial collateral system and bleeding sites are frequently fed by a combination of branches from different territories like the external iliac, contralateral iliac, inferior mesenteric, lumbar, or profunda femoral arteries [33•]. For example, it has been found that the obturator artery has a replaced origin to the external iliac artery in over $30 \%$ of cases; this vessel variant is the so-called corona mortis of pelvic trauma [36]. If the situation allows, at the very least, selective bilateral internal and external iliac angiograms are recommended to identify hemorrhagic arterial branches frequently obscured to the non-selective pelvic angiogram [37•]. Otherwise, proximal embolization of an internal iliac artery, and even empiric embolization of the whole bilateral internal iliac artery system are considered adequate alternatives when multiple branches are affected or when the patients are too unstable for lengthier procedures $[38,39,40 \bullet]$.

Hemorrhage from injury to thoraco-abdominal musculoskeletal arterial branches can contribute to patient morbidity. This is most commonly seen in penetrating trauma. Subselective embolization is an effective therapeutic approach that should be considered. Angiographic localization of the injured vessel is mostly based on CT scan findings. Again, this requires knowledge of the vascular anatomy and operator experience with the embolic materials to be effective in proper vessel selection and accurate embolization, with the goal of achieving as much distal and proximal control of the injured segment. The intercostal, lumbar, circumflex iliac, mammary, and epigastric branches are some of the frequently treated arteries in these cases.

Vascular injuries from trauma that affect the limbs are most frequently managed surgically. Occasionally, there is a role for endovascular management. Embolotherapy can be used to treat a non-essential limb vessel associated with hemorrhage or fistula, such as a single tibio-peroneal runoff artery, if the others are patent, or a small branch of the profunda femoral artery. A stent-graft can be used to treat fistulas, dissections or psedoaneurysms in larger limb vessels like a subclavian artery or a femoro-popliteal artery; however, the limited patency of stent grafts in these size vessels have to be weighed against the risk and benefits of a surgical repair for each individual case.

\section{Conclusion}

Interventional radiology techniques employed for the management of trauma patients have gained acceptance over the last decades. Although high-level evidence with randomized control trials will be difficult to obtain in the trauma scenario, cohort registries are demonstrating the utility of these 
techniques. However, much debate remains over the best techniques and algorithms.

\section{Compliance with Ethical Standards}

Conflict of Interest Drs. Garza-Berlanga and Lopera declare no conflict of interest relevant to this manuscript.

Human and Animal Rights and Informed Consent This article does not contain any studies with human or animal subjects performed by any of the authors.

\section{References}

Papers of particular interest, published recently, have been highlighted as:

- Of importance

1. Roy-Choudhury SH, Gallacher DJ, Pilmer J, Rankin S, Fowler G, Steers J, et al. Relative threshold of detection of active arterial bleeding: in vitro comparison of MDCT and digital subtraction angiography. AJR Am J Roentgenol. 2007;189(5):W238-46. This article demostrates the importance of adequate injury localizaton on imaging for subsequent selective cannulation in order to identify and treat an injury with an endovascular approach.

2. Melloul EE. Management of severe blunt hepatic injury in the era of computed tomography and transarterial embolization: A systematic review and critical appraisal of the literature. J Trauma Acute Care Surg. 2015;79(3):468-74. This review identifies the risks and benefits of angioembolization in liver trauma.

3. Lopez JMJM. Subcapsular hematoma in blunt splenic injury: A significant predictor of failure of nonoperative management. J Trauma Acute Care Surg. 2015;79(6):957-60. This study identifies the subcapsular splenic hematoma as a singificant independent risk-factor.

4. Rosati CC. Management of splenic trauma: a single institution's 8year experience. Am J Surg. 2015;209(2):308-14.

5. Miller PRPR. Prospective trial of angiography and embolization for all grade III to V blunt splenic injuries: nonoperative management success rate is significantly improved. J Am Coll Surg. 2014;218(4):644-8. Study that demostrates the benefit of angioembolization.

6. Alarhayem AQ, Myers JG, Dent D, Lamus D, Lopera J, Liao L, et al. "Blush at first sight": significance of computed tomographic and angiographic discrepancy in patients with blunt abdominal trauma. Am J Surg. 2015;210(6):1104-10. Discussion 10-1. Study that demonstrates the risk of failing to angiographically identify and treat significant vascular injury seen on CT. It suggests empirical treatment benefits.

7. Fang J-FJF. The CT risk factors for the need of operative treatment in initially hemodynamically stable patients after blunt hepatic trauma. J Trauma. 2006;61(3):547-54.

8. Bhullar ISIS. At first blush: absence of computed tomography contrast extravasation in Grade IV or V adult blunt splenic trauma should not preclude angioembolization. J Trauma Acute Care Surg. 2013;74(1):105-12.
9. Schurr MJ, Fabian TC, Gavant M, Croce MA, Kudsk KA, Minard $\mathrm{G}$, et al. Management of blunt splenic trauma: computed tomographic contrast blush predicts failure of nonoperative management. J Trauma Acute Care Surg. 1995;39(3):507-13.

10. Stassen NANA. Selective nonoperative management of blunt splenic injury: an Eastern Association for the Surgery of Trauma practice management guideline. J Trauma Acute Care Surg. 2012;73(5 suppl 4):S294-300.

11. Duchesne JC, Simmons JD, Schmieg Jr RE, McSwain Jr NE, Bellows CF. Proximal splenic angioembolization does not improve outcomes in treating blunt splenic injuries compared with splenectomy: a cohort analysis. J Trauma. 2008;65(6):1346-51. Discussion 51-3.

12. Miller PR, Chang MC, Hoth JJ, Mowery NT, Hildreth AN, Martin RS, et al. Prospective trial of angiography and embolization for all grade III to V blunt splenic injuries: nonoperative management success rate is significantly improved. J Am Coll Surg. 2014;218(4):644-8. Study that demostrates the benefit of angioembolization in redusing risk for NOM failure.

13. Schnuriger B, Inaba K, Konstantinidis A, Lustenberger T, Chan LS, Demetriades D. Outcomes of proximal versus distal splenic artery embolization after trauma: a systematic review and meta-analysis. J Trauma. 2011;70(1):252-60.

14. Dasgupta N, Matsumoto AH, Arslan B, Turba UC, Sabri S, Angle JF. Embolization therapy for traumatic splenic lacerations. Cardiovasc Intervent Radiol. 2012;35(4):795-806.

15. Tominaga GTGT. Immunologic function after splenic embolization, is there a difference? J Trauma. 2009;67(2):289-95.

16. Sclafani SJ, Shaftan GW, Scalea TM, Patterson LA, Kohl L, Kantor A, et al. Nonoperative salvage of computed tomography-diagnosed splenic injuries: utilization of angiography for triage and embolization for hemostasis. J Trauma. 1995;39(5):818-25. Discussion 26-7.

17. Prichayudh SS. Management of liver injuries: predictors for the need of operation and damage control surgery. Injury. 2014;45(9): 1373-7.

18. Demetriades DD. Selective nonoperative management of penetrating abdominal solid organ injuries. Ann Surg. 2006;244(4):620-8.

19. Starling SVSV. Isolated liver gunshot injuries: nonoperative management is feasible? Rev Col Bras Cir. 2015;42(4):238-43.

20. Letoublon CC. Hepatic arterial embolization in the management of blunt hepatic trauma: indications and complications. J Trauma. 2011;70(5):1032-7. Review that proves benefit of angioembolization and states the ischemic risks and need to minimize the treatment as much as possible.

21. Tzeng WS, Wu RH, Chang JM, Lin CY, Koay LB, Uen YH, et al. Transcatheter arterial embolization for hemorrhage caused by injury of the hepatic artery. J Gastroenterol Hepatol. 2005;20(7):1062-8.

22. Dabbs DN, Stein DM, Scalea TM. Major hepatic necrosis: a common complication after angioembolization for treatment of highgrade liver injuries. J Trauma. 2009;66(3):621-7. Discussion 7-9. Review that proves benefit of angioembolization and states the ischemic risks and need to minimize the treatment as much as possible.

23. Stassen NANA. Nonoperative management of blunt hepatic injury: an Eastern Association for the Surgery of Trauma practice management guideline. J Trauma Acute Care Surgery. 2012;73(5 suppl 4): S288-S93.

24. Bryk DJDJ. Guideline of guidelines: a review of urological trauma guidelines. BJU Int. 2016;117(2):226-34.

25. Vozianov SS. Experience of renal artery embolization in patients with blunt kidney trauma. Cen Eur J Urol. 2015;68(4):471-7.

26. Melnitchouk S, Pfammatter T, Kadner A, Dave H, Witzke H, Trentz $\mathrm{O}$, et al. Emergency stent-graft placement for hemorrhage control in acute thoracic aortic rupture. Eur J Cardiothorac Surg. 2004;25(6): 1032-8. 
27. Reed AB, Thompson JK, Crafton CJ, Delvecchio C, Giglia JS. Timing of endovascular repair of blunt traumatic thoracic aortic transections. J Vasc Surg. 2006;43(4):684-8.

28. Xenos ES, Minion DJ, Davenport DL, Hamdallah O, Abedi NN, Sorial EE, et al. Endovascular versus open repair for descending thoracic aortic rupture: institutional experience and meta-analysis. Eur J Cardiothorac Surg. 2009;35(2):282-6.

29. Czermak BV, Waldenberger P, Perkmann R, Rieger M, Steingruber IE, Mallouhi A, et al. Placement of endovascular stent-grafts for emergency treatment of acute disease of the descending thoracic aorta. AJR Am J Roentgenol. 2002;179(2):337-45.

30. Papakostidis C, Kanakaris N, Dimitriou R, Giannoudis PV. The role of arterial embolization in controlling pelvic fracture haemorrhage: a systematic review of the literature. Eur J Radiol. 2012;81(5):897904. Review that demonstrates the benefit of angioembolizaiton in pelvic trauma.

31. Tai DK, Li WH, Lee KY, Cheng M, Lee KB, Tang LF, et al. Retroperitoneal pelvic packing in the management of hemodynamically unstable pelvic fractures: a level I trauma center experience. J Trauma. 2011;71(4):E79-86.

32. Eastridge BJ, Starr A, Minei JP, O'Keefe GE, Scalea TM. The importance of fracture pattern in guiding therapeutic decisionmaking in patients with hemorrhagic shock and pelvic ring disruptions. J Trauma. 2002;53(3):446-50. Discussion 50-1. Proves the concept of uncontrolled hemorrage in pelvic fractures.

33. Lustenberger T, Wutzler S, Stormann P, Laurer H, Marzi I. The role of angio-embolization in the acute treatment concept of severe pelvic ring injuries. Injury. 2015;46 Suppl 4:S33-8. States the benefit of angioembolization in the acute management of unstable pelvic fractures.
34. Cothren CC, Moore EE, Smith WR, Morgan SJ. Preperitoneal pelvic packing in the child with an unstable pelvis: a novel approach. J Pediatr Surg. 2006;41(4):e17-9.

35. Baque P, Trojani C, Delotte J, Sejor E, Senni-Buratti M, de Baque F, et al. Anatomical consequences of "open-book" pelvic ring disruption: a cadaver experimental study. Surg Radiol Anat. 2005;27(6): 487-90.

36. Smith JC, Gregorius JC, Breazeale BH, Watkins GE. The corona mortis, a frequent vascular variant susceptible to blunt pelvic trauma: identification at routine multidetector CT. J Vasc Interv Radiol. 2009;20(4):455-60.

37. Johnson GE, Sandstrom CK, Kogut MJ, Ingraham CR, Stratil PG, Valji K, et al. Frequency of external iliac artery branch injury in blunt trauma: improved detection with selective external iliac angiography. J Vasc Interv Radiol. 2013;24(3):363-9. States the importance of selective bilateral angiograms to adequately identify injury and the benefit of angioembolization.

38. Velmahos GC, Chahwan S, Hanks SE, Murray JA, Berne TV, Asensio J, et al. Angiographic embolization of bilateral internal iliac arteries to control life-threatening hemorrhage after blunt trauma to the pelvis. Am Surg. 2000;66(9):858-62.

39. Ramirez JI, Velmahos GC, Best CR, Chan LS, Demetriades D. Male sexual function after bilateral internal iliac artery embolization for pelvic fracture. J Trauma. 2004;56(4):7349. Discussion 9-41.

40. Travis T, Monsky WL, London J, Danielson M, Brock J, Wegelin J, et al. Evaluation of short-term and long-term complications after emergent internal iliac artery embolization in patients with pelvic trauma. J Vasc Interv Radiol. 2008;19(6):840-7. Efficacy and safety of iliac artery embolization. 